\title{
EXTENSIONS AND AMPLIFICATIONS OF A TRAFFIC MODEL OF AW AND RASCLE*
}

\author{
J. M. GREENBERG ${ }^{\dagger}$
}

Abstract. In a recent paper Aw and Rascle [SIAM J. Appl. Math., 60 (2000), pp. 916-938] introduced a new model of traffic on a uni-directional highway. Here the author studies an extension of this model, one which accounts for drivers attempting to travel at the maximum allowable speed. The author looks at a Lagrangian reformulation of this problem, a formulation that leads to an effective computational algorithm for solving the resulting system.

Key words. conservation laws, hyperbolic equations, traffic flow, relaxation model

AMS subject classification. 35

PII. S0036139900378657

1. Introduction. In a recent paper Aw and Rascle [1] introduced a new phenomenological model for traffic on a uni-directional roadway. The basic descriptors of their model are the density $\rho(x, t) \geq 0$ and velocity $u(x, t) \geq 0$ of cars located at position $x$ at time $t$. In the classic traffic model studied by Lighthill, Whitham, and Richards (see [2], [3], and [4]), the velocity $u$ is given in terms of $\rho$ by

$$
u=v(\rho),
$$

where $\rho \rightarrow v(\rho)$ is a smooth, nonnegative, strictly decreasing function on $\left[0, \rho_{\max }\right]$ satisfying

$$
v\left(\rho_{\max }\right)=0
$$

In what follows we refer to this as the LWR model. For the LWR model, the governing equation is the continuity equation

$$
\frac{\partial \rho}{\partial t}+\frac{\partial}{\partial x}(\rho v(\rho))=0
$$

These authors make the additional assumption that the flux, $F(\rho) \stackrel{\text { def }}{=} \rho v(\rho)$, which satisfies $0=F(0)=F\left(\rho_{\max }\right)$, is strictly concave on $\left[0, \rho_{\max }\right]$, i.e.,

$$
\frac{d^{2} F}{d \rho^{2}}=2 v^{\prime}(\rho)+\rho v^{\prime \prime}(\rho)<0, \quad 0 \leq \rho \leq \rho_{\max } .
$$

They solve (1.3) subject to suitable initial and boundary conditions which guarantee that the solution satisfies $0 \leq \rho(x, t) \leq \rho_{\max }$. We shall also assume that (1.4) holds.

Aw and Rascle allow for any car velocity $u$ satisfying

$$
0 \leq u \leq v(\rho)
$$

*Received by the editors September 26, 2000; accepted for publication (in revised form) March 8, 2001; published electronically December 28, 2001. This research was partially supported by the Applied Mathematical Sciences Program, U.S. Department of Energy, and the Mathematics and Computer Science Division, Army Research Office.

http://www.siam.org/journals/siap/62-3/37865.html

${ }^{\dagger}$ Department of Mathematical Sciences, Carnegie Mellon University, Pittsburgh, PA 15213 (greenber@andrew.cmu.edu). 
where $v(\cdot)$ is in the LWR model. Their governing balance law is again the continuity equation

$$
\frac{\partial \rho}{\partial t}+\frac{\partial}{\partial x}(\rho u)=0
$$

and they close their system by assuming that $\alpha \stackrel{\text { def }}{=} u-v(\rho) \in[-v(0), 0]$ passively advects with velocity $u$, i.e., satisfies

$$
\frac{\partial \alpha}{\partial t}+u \frac{\partial \alpha}{\partial x}=0
$$

They interpret (1.7) as the Lagrangian form of the conservation law

$$
\frac{\partial}{\partial t}(\rho(u-v(\rho)))+\frac{\partial}{\partial x}(\rho u(u-v(\rho)))=0,
$$

but this latter interpretation is unnecessary. Aw and Rascle note that smooth solutions of (1.6) and (1.7) also satisfy

$$
\frac{\partial u}{\partial t}+\left(u+\rho v^{\prime}(\rho)\right) \frac{\partial u}{\partial x}=0,
$$

and this latter system, namely (1.7) and (1.9), is strictly hyperbolic with wave speeds $c^{1}$ and $c^{2}$ satisfying

$$
c^{1} \stackrel{\text { def }}{=} u+\rho v^{\prime}(\rho)=\alpha+F^{\prime}(\rho)<c^{2} \stackrel{\text { def }}{=} u
$$

so long as $0<\rho<\rho_{\max }$. Once again,

$$
-v(0) \leq \alpha \stackrel{\text { def }}{=} u-v(\rho) \leq 0 \text { and } F(\rho) \stackrel{\text { def }}{=} \rho v(\rho) .
$$

Discontinuous solutions of (1.6) and (1.7) satisfy the Rankine-Hugoniot equation associated with (1.6), namely

$$
\frac{d s}{d t}[\rho]=[u \rho]=\frac{\left(\rho_{-}+\rho_{+}\right)}{2}[u]+\frac{\left(u_{-}+u_{+}\right)}{2}[\rho] .
$$

Here $x=s(t), t_{1}<t<t_{2}$, is a curve across which the solution of (1.6) and (1.7) experiences a jump discontinuity, $\left(\rho_{-}, u_{-}\right)(t) \stackrel{\text { def }}{=} \lim _{\epsilon \rightarrow 0^{+}}(\rho, u)(s(t)-\epsilon, t),\left(\rho_{+}, u_{+}\right)(t) \stackrel{\text { def }}{=}$ $\lim _{\epsilon \rightarrow 0^{+}}(\rho, u)(s(t)+\epsilon, t)$, and finally $[\rho]=\rho_{-}-\rho_{+}$and $[u]=u_{-}-u_{+}$.

Discontinuities associated with the system (1.6) and (1.7) come in two flavors. The first type, called contact discontinuities, have the property that $[u]=0$ and $\frac{d s}{d t}=u_{-}=u_{+}$. In this situation, (1.12) supplies no information about $[\rho]$, but (1.7) implies that

$$
v\left(\rho_{-}\right)=v\left(\rho_{+}\right)+\alpha_{+}-\alpha_{-}
$$

The fact that

$$
u_{+}=u_{-}>c_{-}^{1}=\alpha_{-}+F^{\prime}\left(\rho_{-}\right)=\alpha_{-}+v\left(\rho_{-}\right)+\rho_{-} v^{\prime}\left(\rho_{-}\right)
$$

implies that $\rho_{-}$is determined by the $\rho_{+}$and the states $\alpha_{+}$and $\alpha_{-}$, which, by virtue of (1.7), are given in terms of the initial data by

$$
\alpha_{-}(t)=\alpha_{-}(0)=\lim _{\epsilon \rightarrow 0^{+}} \alpha\left(s(0)-\epsilon, 0^{+}\right)
$$


and

$$
\alpha_{+}(t)=\alpha_{+}(0)=\lim _{\epsilon \rightarrow 0^{+}} \alpha\left(s(0)+\epsilon, 0^{+}\right) .
$$

This last observation was apparently not noticed by Aw and Rascle, but it does point out the anticipatory nature of the system, namely that $u$ and $\rho$ behind a contact are determined by $u$ and $\rho$ ahead of it. This observation is exploited in section 2 with our downwind difference scheme.

In the second family of discontinuities we have

$$
\alpha_{-} \stackrel{\text { def }}{=} u_{-}-v\left(\rho_{-}\right)=u_{+}-v\left(\rho_{+}\right) \stackrel{\text { def }}{=} \alpha_{+} .
$$

This latter relationship, when combined with (1.12), implies that

$$
\frac{d s}{d t}[\rho]=\alpha_{-}[\rho]+[F(\rho)]
$$

or

$$
\frac{d s}{d t}=\alpha_{-}+\frac{[F(\rho)]}{[\rho]}
$$

Once again $F(\rho) \stackrel{\text { def }}{=} \rho v(\rho)$ and we assume that $F$ satisfies (1.4). For such discontinuities to be admissible we insist that $0 \leq \rho_{-}<\rho_{+} \leq \rho_{\max }$. This admissibility condition guarantees that the Lax entropy criterion

$$
c_{-}^{1} \stackrel{\text { def }}{=} \alpha_{-}+F^{\prime}\left(\rho_{-}\right)>\dot{s}=\alpha_{-}+\frac{\left(F\left(\rho_{-}\right)-F\left(\rho_{+}\right)\right)}{\rho_{-}-\rho_{+}}>c_{+}^{1} \stackrel{\text { def }}{=} \alpha_{+}+F^{\prime}\left(\rho_{+}\right)
$$

is satisfied across these discontinuities.

One weakness of the Aw-Rascle model is the fact that drivers operating at speed $u$ in traffic with local density $\rho$ never adjust their vehicles' operation to achieve the maximum allowable speed, namely $v(\rho)$. Here we shall account for that and replace (1.7) by

$$
\frac{\partial}{\partial t}(u-v(\rho))+u \frac{\partial}{\partial x}(u-v(\rho))=-\frac{(u-v(\rho))}{\delta},
$$

where $0<\delta$ is interpreted as a relaxation or adjustment time. Our model has the same discontinuity structure as the $\mathrm{Aw}$-Rascle model. The only difference is that across contact discontinuities $\alpha_{-}(t)=e^{-t / \delta} \alpha_{-}(0)$ and $\alpha_{+}(t)=e^{-t / \delta} \alpha_{+}(0)$, where $\left(\alpha_{-}, \alpha_{+}\right)(0)=\lim _{\epsilon \rightarrow 0^{+}}\left(\alpha\left(s(0)-\epsilon, 0^{+}\right), \alpha\left(s(0)+\epsilon, 0^{-}\right)\right)$.

Our goal is an effective computational scheme for solving (1.6) and (1.21). In section 2 we present such a scheme. It is based on a Lagrangian reformulation of the system and leads naturally to a downwind integration scheme which is stable and well behaved. This scheme exploits the fact that the system has no mechanism to spontaneously generate contact discontinuities but does require additional smoothness in the field $\alpha=u-v(\rho)$ at $t=0$.

In section 3 we present some interesting numerical simulations.

Throughout, we restrict our attention to initial data $u^{0}(\cdot)$ and $\rho^{0}(\cdot)$ satisfying

$$
0 \leq u^{0}(x) \leq v\left(\rho^{0}(x)\right)
$$


whenever $\rho^{0}(x)>0$. One consequence of our analysis is that for future times these inequalities are preserved; that is,

$$
0 \leq u(x, t) \leq v(\rho(x, t))
$$

whenever $\rho(x, t)>0$. This assumption on the data was criticized by one reviewer and we justify it by saying that our highway is populated by enlightened individuals who drive safely. It was by no means obvious for the relaxation models that if the drivers were enlightened at $t=0$, then they would stay enlightened for all future times but, in fact, this is the case.

2. Lagrangian reformulation and scheme I. Our first computational model for the system (1.6) and (1.21) is based on an equivalent Lagrangian formulation. To keep things simple we assume that the initial data satisfies

$$
-v(\epsilon) \leq \alpha^{0}(x) \leq 0
$$

and

$$
\epsilon \leq \rho^{0}(x) \leq v^{-1}\left(-\alpha^{0}(x)\right)
$$

for $-L<x<L$. If $L<\infty$ we can allow $\rho^{0}(x) \equiv 0$ for $|x|>L$. In this situation, $\alpha^{0}(x)$ is unspecified for $|x|>L$.

We let

$$
M(x) \stackrel{\text { def }}{=} \int_{0}^{x} \frac{\rho_{0}(\xi)}{\rho_{\max }} d \xi, \quad-\infty<x<\infty,
$$

define the constants $M_{-}$and $M_{+}$by

$$
M_{-}=\lim _{x \rightarrow-\infty} M(x) \text { and } M_{+}=\lim _{x \rightarrow \infty} M(x),
$$

and for any $M \in\left(M_{-}, M_{+}\right)$we let $\chi_{0}(M)$ be the unique solution of

$$
M=\int_{0}^{\chi_{0}(M)} \frac{\rho_{0}(\xi)}{\rho_{\max }} d \xi .
$$

We further suppose that $(\rho, u, \alpha)$ is a solution of the initial value problem

$$
\begin{gathered}
\frac{\partial \rho}{\partial t}+\frac{\partial}{\partial x}(\rho u)=0, \\
\frac{\partial \alpha}{\partial t}+u \frac{\partial \alpha}{\partial x}=\frac{-\alpha}{\delta}, \\
u=v(\rho)+\alpha,
\end{gathered}
$$

and

$$
\rho\left(x, 0^{+}\right)=\rho^{0}(x) \text { and } \alpha\left(x, 0^{+}\right)=\alpha^{0}(x), \quad-\infty<x<\infty ;
$$

let $x=\chi(M, t)$ be the autotrajectory defined by

$$
\frac{\partial \chi}{\partial t}=v(\rho(\chi, t))+\alpha(\chi, t) \text { and } \chi\left(M, 0^{+}\right)=\chi_{0}(M) ;
$$


and observe that (2.5), (2.6), and (2.10) imply that for any

$$
\begin{aligned}
& M_{-}<M_{1}<M_{2}<M_{+}, \\
& M_{2}-M_{1}=\int_{\chi\left(M_{1}, t\right)}^{\chi\left(M_{2}, t\right)} \frac{\rho(x, t)}{\rho_{\max }} d x .
\end{aligned}
$$

This latter identity in turn yields

$$
\gamma(M, t) \rho(\chi(M, t), t) \equiv \rho_{\max },
$$

where

$$
\gamma(M, t)=\frac{\partial \chi}{\partial M}(M, t) .^{1}
$$

Moreover, if we let

$$
A(M, t) \stackrel{\text { def }}{=} \alpha(\chi(M, t), t)
$$

then (2.7), (2.8), and (2.10) imply that $A$ satisfies

$$
\frac{\partial A}{\partial t}=-\frac{A}{\delta}, \quad M_{-}<M<M_{+}
$$

while (2.10) and (2.13) imply that

$$
\frac{\partial \gamma}{\partial t}=\frac{\partial}{\partial M}(\tilde{v}(\gamma)+A), \quad M_{-}<M<M_{+} .
$$

Here

$$
\tilde{v}(\gamma)=v\left(\frac{\rho_{\max }}{\gamma}\right)
$$

and $\rho \rightarrow v(\rho)$ is the function introduced in (1.1). The system (2.15) and (2.16) is solved subject to the initial conditions

$$
A\left(M, 0^{+}\right)=\alpha^{0}\left(\chi_{0}(M)\right) \text { and } \gamma\left(M, 0^{+}\right)=\frac{\partial \chi_{0}}{\partial M}(M)=\frac{\rho_{\max }}{\rho^{0}\left(\chi^{0}(M)\right)}, M_{-}<M<M_{+},
$$

where, once again, $\chi_{0}(\cdot)$ is defined in (2.5). We note that the hypotheses $v^{\prime}(\rho)<0$ and $\left(\rho v^{\prime \prime}(\rho)+2 v^{\prime}(\rho)\right)<0$ imply that

$$
\frac{\partial \tilde{v}}{\partial \gamma}=-\frac{\rho_{\max }}{\gamma^{2}} v^{\prime}\left(\frac{\rho_{\max }}{\gamma}\right)>0, \quad \gamma \geq 1
$$

and

$$
\frac{\partial^{2} \tilde{v}}{\partial \gamma^{2}}=\frac{\rho_{\max }}{\gamma^{3}}\left(\frac{\rho_{\max }}{\gamma} v^{\prime \prime}\left(\frac{\rho_{\max }}{\gamma}\right)+2 v^{\prime}\left(\frac{\rho_{\max }}{\gamma}\right)\right)<0
$$

\footnotetext{
${ }^{1}$ This is a standard reformulation in gas dynamics; for details, see Courant and Friedrichs [5, p. 30].
} 
Computational model. Our computational model is based on the Lagrangian system (2.15) and (2.16). The fact that $\frac{\partial \tilde{v}}{\partial \gamma}>0$ suggests using downwind differencing in (2.16). Such differencing is, in fact, equivalent to applying a first order Godunov scheme to (2.16). Our spatial grid has points

$$
M_{k} \stackrel{\text { def }}{=} \int_{0}^{x_{k}^{0}} \frac{\rho^{0}(\xi)}{\rho_{\max }} d \xi
$$

and $0<(\Delta M)_{k}=M_{k+1}-M_{k}$.

In what follows, $\gamma_{k}^{n}$ will denote the cell average of $\gamma(\cdot, n \Delta t)$ over $\left(M_{k}, M_{k+1}\right)$,

$$
(\Delta M)_{k} \gamma_{k}^{n}=\int_{M_{k}}^{M_{k+1}} \gamma(M, n \Delta t) d M
$$

and $A_{k}^{n}$ will denote the point value

$$
A_{k}^{n}=A\left(M_{k}, n \Delta t\right)
$$

We replace (2.16) with the downwind difference approximation

$$
\gamma_{k}^{n+1}=\gamma_{k}^{n}+\frac{\Delta t}{(\Delta M)_{k}}\left(\tilde{v}\left(\gamma_{k+1}^{n}\right)+A_{k+1}^{n}-\tilde{v}\left(\gamma_{k}^{n}\right)-A_{k}^{n}\right)
$$

and (2.17) with

$$
A_{k}^{n}=A_{k}^{0} e^{-n \Delta t / \delta}=\alpha^{0}\left(\chi_{0}\left(M_{k}\right)\right) e^{-n \Delta t / \delta} .
$$

The trajectory updates are given by

$$
x_{k}^{n+1}=x_{k}^{n}+\Delta t\left(\tilde{v}\left(\gamma_{k}^{n}\right)+A_{k}^{n}\right) \text {, }
$$

and these satisfy

$$
\frac{x_{k+1}^{n+1}-x_{k}^{n+1}}{(\Delta M)_{k}}=\gamma_{k}^{n+1}
$$

For completeness, we also update the density by

$$
\left(x_{k+1}^{n+1}-x_{k}^{n+1}\right) \rho_{k}^{n+1}=\rho_{\max }(\Delta M)_{k} .
$$

The numbers $\rho_{k}^{n+1}$ are the cell averages of the density over the interval $\left(x_{k}^{n+1}, x_{k+1}^{n+1}\right)$.

Up to this point we have said nothing about boundary conditions. Based on (2.2) we know that $\rho^{0}(x) \geq \epsilon$ on $(-L, L)$. If $L=+\infty$, then no boundary conditions are necessary. If $L<\infty$ and $\rho^{0} \equiv 0, x>L$, then

$$
M_{+}=\int_{0}^{L} \frac{\rho^{0}(\xi)}{\rho_{\max }} d \xi<\infty .
$$

In this situation, we impose the boundary condition

$$
\frac{\partial \chi}{\partial t}\left(M_{+}, t\right)=v(0)+A_{-}\left(M_{+}, t\right)=v(0)+\alpha_{-}^{0}(L) e^{-t / \delta}
$$


or its discrete analogue

$$
x_{N+1}^{n+1}=x_{N+1}^{n}+\Delta t\left(v(0)+\alpha_{-}^{0}(L) e^{-n \Delta t / \delta}\right) .
$$

Here, the index $(N+1)$ is such that $M_{N+1}=M_{+}=\int_{0}^{L} \frac{\rho^{0}(\xi)}{\rho_{\max }} d \xi$. In the region $x>\chi\left(M_{+}, t\right)$ the density will be zero; i.e., no cars are present. The situation where $\rho^{0}(x) \equiv 0$ for $x<L$ is handled differently. No boundary condition is required. This is because of the positivity of $\frac{\partial \tilde{v}}{\partial \gamma}$. If $N_{1}<0$ is the index so that

$$
-\infty<M_{N_{1}}=\int_{0}^{-L} \frac{\rho^{0}(\xi)}{\rho} \stackrel{d e f}{=} M_{-}<0,
$$

then we use the standard updates (2.24) for this index. Of course, the actual solution has the property that $\rho(x, t) \equiv 0$ for $x<\chi\left(M_{-}, t\right)$.

Estimates for (2.24) and (2.25). We note that (2.17) and (2.20) imply that

$$
\max _{\gamma \geq 1}\left(\frac{\partial \tilde{v}}{\partial \gamma}(\gamma)\right)=\frac{\partial \tilde{v}}{\partial \gamma}(1)=\rho_{\max }\left|v^{\prime}\left(\rho_{\max }\right)\right|
$$

In what follows, we shall assume that $\Delta t$ is such that

$$
\frac{\rho_{\max }\left|v^{\prime}\left(\rho_{\max }\right)\right|}{(\Delta M)_{k}} \Delta t<1
$$

Lemma 2.1. Suppose that for all $k$ in our computational index set

$$
\gamma_{k}^{n} \geq 1, \quad 0 \leq \tilde{v}\left(\gamma_{k}^{n}\right)+A_{k}^{n}, \quad A_{k}^{n} \leq 0,
$$

and that (2.31) holds. Then the same inequalities hold for index $n+1$.

Proof. We first note that if $\tilde{v}\left(\gamma_{k}^{n}\right)+A_{k+1}^{n}-A_{k}^{n} \geq \lim _{\gamma \rightarrow \infty} \tilde{v}(\gamma)=v(0)$, then (2.24) implies that $\gamma_{k}^{n+1} \geq \gamma_{k}^{n} \geq 1$. On the other hand, if $\tilde{v}\left(\gamma_{k}^{n}\right)+A_{k+1}^{n}-A_{k}^{n}<v(0)$, then (2.32) implies that there exists a unique $1 \leq \Gamma_{k}^{n}<\infty$ such that $\tilde{v}\left(\Gamma_{k}^{n}\right)=\tilde{v}\left(\gamma_{k+1}^{n}\right)+$ $A_{k+1}^{n}-A_{k}^{n}$, and this latter identity, when combined with (2.24) and (2.31), implies that $1 \leq \min \left(\Gamma_{k}^{n}, \gamma_{k}^{n}\right) \leq \gamma_{k}^{n+1} \leq \max \left(\Gamma_{k}^{n}, \gamma_{k}^{n}\right)$. We now turn to establishing the inequality $\tilde{v}\left(\gamma_{k}^{n+1}\right)+A_{k}^{n+1} \geq 0$. If $\tilde{v}\left(\gamma_{k+1}^{n}\right)+A_{k+1}^{n}-A_{k}^{n} \geq v(0)$, then the inequality $\gamma_{k}^{n+1} \geq \gamma_{k}^{n} \geq 1$ implies that $\tilde{v}\left(\gamma_{k}^{n+1}\right)+A_{k}^{n+1} \geq \tilde{v}\left(\gamma_{k}^{n}\right)+A_{k}^{n}+A_{k}^{n+1}-A_{k}^{n}$, and our induction hypothesis $\tilde{v}\left(\gamma_{k}^{n}\right)+A_{k}^{n} \geq 0$, together with $A_{k}^{n+1}-A_{k}^{n}=\left(e^{-\Delta t / \delta}-1\right) A_{k}^{n} \geq 0$, yields the desired result. On the other hand, if $\tilde{v}\left(\gamma_{k+1}^{n}\right)+A_{k+1}^{n}-A_{k}^{n}<v(0)$, then $\tilde{v}\left(\Gamma_{k}^{n}\right)=\tilde{v}\left(\gamma_{k+1}^{n}\right)+A_{k+1}^{n}-A_{k}^{n}$ and $\min \left(\Gamma_{k}^{n}, \gamma_{k}^{n}\right) \leq \gamma_{k}^{n+1} \leq \max \left(\Gamma_{k}^{n}, \gamma_{k}^{n}\right)$. If $\Gamma_{k}^{n}<\gamma_{k}^{n}$, then $\tilde{v}\left(\gamma_{k}^{n+1}\right)+A_{k}^{n+1} \geq \tilde{v}\left(\Gamma_{k}^{n}\right)+A_{k}^{n+1}=\left(\tilde{v}\left(\gamma_{k+1}^{n}\right)+A_{k+1}^{n}\right)+\left(A_{k}^{n+1}-A_{k}^{n}\right) \geq 0$. On the other hand, if $\gamma_{k}^{n} \leq \Gamma_{k}^{n}$, then $\tilde{v}\left(\gamma_{k}^{n+1}\right)+A_{k}^{n+1} \geq\left(\tilde{v}\left(\gamma_{k}^{n}\right)+A_{k}\right)+\left(A_{k}^{n+1}-A_{k}^{n}\right) \geq 0$, and this completes the proof.

The following lemma covers the situation where the initial data satisfies $\rho^{0}(x) \geq$ $\epsilon>0$ on $-\infty<x<\infty$. This hypothesis, together with (2.18), guarantees that the initial sequence satisfies $\gamma_{k}^{0} \leq \frac{\rho_{\max }}{\epsilon}$.

LEMma 2.2. If $\rho^{0}(x) \geq \epsilon>0$ on $-\infty<x<\infty$ and (2.31) holds, then the sequence $\left\{\gamma_{k}^{n}\right\}$ defined in (2.24) satisfies

$$
\gamma_{k}^{n} \leq \frac{\rho_{\max }}{\epsilon}\left[1+\max _{\infty<x<\infty}\left|\alpha_{x}^{0}(x)\right| \frac{\Delta t}{1-e^{-\Delta t / \delta}}\right] .
$$


Proof. The identities (2.24) and (2.25) imply that

$$
\begin{aligned}
\gamma_{k}^{n+1}= & \left(1-\frac{\Delta t c_{k, k+1}^{n}}{(\Delta M)_{k}}\right) \gamma_{k}^{n}+\frac{\Delta t c_{k, k+1}^{n}}{(\Delta M)_{k}} \gamma_{k+1}^{n} \\
& +\Delta t\left(\frac{\alpha^{0}\left(\chi_{0}\left(M_{k+1}\right)\right)-\alpha^{0}\left(\chi_{0}\left(M_{k}\right)\right)}{\chi_{0}\left(M_{k+1}\right)-\chi_{0}\left(M_{k}\right)}\right)\left(\frac{\chi_{0}\left(M_{k+1}\right)-\chi_{0}\left(M_{k}\right)}{(\Delta M)_{k}}\right) e^{-n \Delta t / \delta},
\end{aligned}
$$

where $0 \leq c_{k, k+1}^{n}=\frac{\tilde{v}\left(\gamma_{k+1}^{n}\right)-\tilde{v}\left(\gamma_{k}^{n}\right)}{\gamma_{k+1}^{n}-\gamma_{k}^{n}} \leq \rho_{\max }\left|v^{\prime}\left(\rho_{\max }\right)\right|$. Moreover, (2.31) implies that $0 \leq \frac{\Delta t c_{k, k+1}^{n}}{(\Delta M)_{k}} \leq 1$, and thus (2.34) when combined with $\frac{\partial \chi_{0}}{\partial M} \leq \frac{\rho_{\max }}{\epsilon}$ implies that $\Gamma^{n} \stackrel{\text { def }}{=} \max _{k}\left(\gamma_{k}^{n}\right)$ satisfies

$$
\Gamma^{n+1} \leq \Gamma^{n}+\Delta t \frac{\rho_{\max }}{\epsilon} \max _{\infty<x<\infty}\left|\alpha_{x}^{0}(x)\right| e^{-n \Delta t / \delta},
$$

and (2.35) and $\Gamma^{0} \leq \frac{\rho_{\max }}{\epsilon}$ then yield the desired upper bound.

We note that the estimates

$$
1 \leq \gamma_{k}^{n} \leq \frac{\rho_{\max }}{\epsilon}\left[1+\max _{-\infty<x<\infty}\left|\alpha_{x}^{0}(x)\right| \frac{\Delta t}{\left(1-e^{-\Delta t / \delta}\right)}\right]
$$

when combined with (2.28), imply that

$$
\frac{\epsilon}{\left[1+\max _{-\infty<x<\infty}\left|\alpha_{x}^{0}(x)\right| \frac{\Delta t}{\left(1-e^{-\Delta t / \delta}\right)}\right]} \leq \rho_{k}^{n} \leq \rho_{\max } .
$$

In the situation where $L<\infty$ and $\rho^{0}(x) \equiv 0, x>L$, we lose the upper bound (2.36). The bound fails because for the index $k=N$, where $M_{N+1}=M_{+}=\int_{0}^{L} \frac{\rho^{0}(\xi)}{\rho_{\max }} d \xi$, we have

$$
\gamma_{N}^{n+1}=\gamma_{N}^{n}+\frac{\Delta t}{(\Delta M)_{N}}\left[\tilde{v}(\infty)-\tilde{v}\left(\gamma_{k}^{n}\right)+\left(\alpha_{-}^{0}(L)-\alpha^{0}\left(\chi_{0}\left(M_{N}\right)\right)\right) e^{-n \Delta t / \delta}\right],
$$

and the argument used to establish Lemma 2.2 fails. In this situation we are left with the estimates

$$
1 \leq \gamma_{k}^{n}, \quad 0 \leq \tilde{v}\left(\gamma_{k}^{n}\right)+A_{k}^{n}, \quad \text { and } 0 \leq \rho_{k}^{n} \leq \rho_{\max }
$$

Our final estimate supplies a one sided bound for $\frac{\partial \gamma}{\partial M}$. Here we require that the points $\left\{x_{k}^{0}\right\}$ and $\left\{M_{k}\right\}$ defined by

$$
M_{k}=\int_{0}^{x_{k}^{0}} \frac{\rho^{0}(\xi)}{\rho_{\max }} d \xi
$$

satisfy

$$
\int_{x_{k}^{0}}^{x_{k+1}^{0}} \frac{\rho^{0}(\xi)}{\rho_{\max }} d \xi \equiv \Delta M, \text { a constant. }
$$

We also require that $\rho^{0}(x) \geq \epsilon>0$ on $(-\infty, \infty)$. 
LEMMA 2.3. If the hypotheses of the preceding paragraph hold, and if (2.31) is valid, then

$$
\begin{aligned}
\max _{k}\left(\max \left[0, \frac{\gamma_{k+1}^{n}-\gamma_{k}^{n}}{\Delta M}\right]\right) & \leq \max _{k}\left(\max \left[0, \frac{\gamma_{k+1}^{0}-\gamma_{k}^{0}}{\Delta M}\right]\right) \\
& +\max _{M}\left|\frac{\partial^{2} \alpha^{0}\left(\chi_{0}(M)\right)}{\partial M^{2}}\right| \frac{\Delta t}{1-e^{-\Delta t / \delta}} .
\end{aligned}
$$

Proof. We observe that $(2.24)$ with $(\Delta M)_{k} \equiv \Delta M$ implies that

$$
\begin{aligned}
& \gamma_{k+1}^{n}-\gamma_{k}^{n} \\
& =\left(\gamma_{k+1}^{n-1}-\gamma_{k}^{n-1}\right)+\frac{\Delta t}{\Delta M}\left(\tilde{v}\left(\gamma_{k+2}^{n-1}\right)-\tilde{v}\left(\gamma_{k+1}^{n-1}\right)\right)-\frac{\Delta t}{\Delta M}\left(\tilde{v}\left(\gamma_{k+1}^{n-1}\right)-\tilde{v}\left(\gamma_{k}^{n-1}\right)\right) \\
& +\frac{\Delta t}{\Delta M}\left(\alpha^{0}\left(\chi_{0}\left(M_{k+2}\right)\right)-2 \alpha^{0}\left(\chi_{0}\left(M_{k+1}\right)\right)+\alpha^{0}\left(\chi_{0}\left(M_{k}\right)\right)\right) e^{-(n-1) \Delta t / \delta} \\
& =\left(1-\frac{\Delta t}{\Delta M} \tilde{v}^{\prime}\left(\gamma_{k+1}^{n-1}\right)\right)\left(\gamma_{k+1}^{n-1}-\gamma_{k}^{n-1}\right)+\frac{\Delta t}{\Delta M} \tilde{v}^{\prime}\left(\gamma_{k+1}^{n-1}\right)\left(\gamma_{k+2}^{n-1}-\gamma_{k+1}^{n-1}\right) \\
& +\frac{\Delta t}{2 \Delta M} \tilde{v}^{\prime \prime}\left(\theta_{k, k+1}^{n-1}\right)\left(\gamma_{k+1}^{n-1}-\gamma_{k}^{n-1}\right)^{2}+\frac{\Delta t}{2 \Delta M} \tilde{v}^{\prime \prime}\left(\theta_{k+1, k+2}^{n-1}\right)\left(\gamma_{k+2}^{n-1}-\gamma_{k+1}^{n-1}\right)^{2} \\
& +\frac{\Delta t}{\Delta M}\left(\alpha^{0}\left(\chi_{0}\left(M_{k+2}\right)\right)-2 \alpha^{0}\left(\chi_{0}\left(M_{k+1}\right)\right)+\alpha^{0}\left(\chi_{0}\left(M_{k}\right)\right)\right) e^{-(n-1) \Delta t / \delta} .
\end{aligned}
$$

Moreover, (2.42) and $\tilde{v}^{\prime \prime}<0$ imply that

$$
\begin{aligned}
\max _{k}\left(\max \left[0, \frac{\gamma_{k+1}^{n}-\gamma_{k}^{n}}{\Delta M}\right]\right) & \leq \max _{k}\left(\max \left[0, \frac{\gamma_{k+1}^{n-1}-\gamma_{k}^{n-1}}{\Delta M}\right]\right) \\
& +\Delta t \max _{M}\left|\frac{\partial^{2} \alpha^{0}}{\partial M^{2}}\left(\chi_{0}(M)\right)\right| e^{-(n-1) \Delta t / \delta}
\end{aligned}
$$

and (2.43), together with $\Delta t \sum_{k=0}^{n-1} e^{-k \Delta t / \delta} \leq \frac{\Delta t}{1-e^{-\Delta t / \delta}}$, yields the desired result.

We note that if our time step $\Delta t$ is chosen so that

$$
\frac{\partial \tilde{v}}{\partial \gamma}(1) \frac{\Delta t}{\Delta M}=\rho_{\max }\left|v^{\prime}\left(\rho_{\max }\right)\right| \frac{\Delta t}{\Delta M}=\mu \in(0,1),
$$

then the estimate (2.41) implies that

$$
\begin{aligned}
P V(\gamma(M, n \Delta t)) \leq & P V\left(\gamma\left(M, 0^{+}\right)\right) \\
\left\{M_{1}<M<M_{2}\right\} \leq & \left\{M_{1}<M<M_{2}+\frac{\rho_{\max }\left|v^{\prime}\left(\rho_{\max }\right)\right|}{\mu}(n \Delta t)\right\} \\
& +\max _{M}\left|\frac{\partial^{2} \alpha^{0}\left(\chi_{0}(M)\right)}{\partial M^{2}}\right|\left(M_{2}+\frac{\rho_{\max }\left|v^{\prime}\left(\rho_{\max }\right)\right|}{\mu}(n \Delta t)-M_{1}\right) .
\end{aligned}
$$

Here $P V$ denotes the positive variation. This latter estimate, together with the results of Lemma 2.2, also implies that the total variation of $\gamma(\cdot, n \Delta t)$ over $\left(M_{1}, M_{2}\right)$ is a priori bounded in terms of the initial data, and this latter estimate is sufficient to guarantee that our approximate solutions converge to weak solutions of (2.15), (2.16), and (2.18). The one sided estimate (2.41) also guarantees that our weak solutions satisfy $\gamma_{-}>\gamma_{+}$(equivalently $\rho_{-}<\rho_{+}$) across shocks.

3. Simulations. The computations which we present here were computed using the Lagrangian formulation of section 2; in particular (2.25)-(2.28). The graphical 
displays render the piecewise constant cell averages, $\left\{\rho_{k}^{n}\right\}$, and particle velocities, $u_{k}^{n}=v\left(\rho_{k}^{n}\right)+A_{k}^{n}$, versus the current particle positions $\left\{x_{k}^{n}\right\}$. In these computations we also introduced adaptivity to maintain resolution of the density. In particular, if $\left(x_{k+1}^{n}-x_{k}^{n}\right)$ exceeds a prescribed threshold, then we introduce a new particle $\xi_{k}^{n}=$ $\left(\frac{x_{k}^{n}+x_{k+1}^{n}}{2}\right)$, locally modify $\rho$ and $A$ by

$$
\left(\rho^{\text {new }}\right)^{n}(x)=\rho_{k}^{n}, \quad x_{k}^{n}<x<x_{k+1}^{n} \text { and }\left(\alpha^{\text {new }}\right)^{n}(x)= \begin{cases}A_{k}^{n}, & x=x_{k}^{n}, \\ \frac{A_{k}^{n}+A_{k+1}^{n}}{2}, & x=\xi_{k}^{n}, \\ A_{k+1}^{n}, & x=x_{k+1}^{n},\end{cases}
$$

and then perform our update.

All simulations were run with $\delta=1$ and

$$
v(\rho)=1-\rho
$$

or equivalently

$$
\tilde{v}(\gamma)=1-\frac{1}{\gamma}
$$

where

$$
\gamma=1 / \rho
$$

The first simulation is as run with the initial data

$$
\rho^{0}(x)= \begin{cases}0, & x<-1 \\ 4, & -1<x<0 \\ .2, & 0<x<1 \\ 4, & 1<x<2 \\ 0, & 2<x\end{cases}
$$

and

$$
\alpha^{0}(x)= \begin{cases}0, & x<-1 \\ -\left(\sin ^{2}(\pi x)\right) / 3, & -1 \leq x \leq 2, \\ 0, & 1<x\end{cases}
$$

At $t=0$ our initial distribution of grid points was

$$
x_{k}^{0}= \begin{cases}-1+\frac{k}{400}, & 0 \leq k \leq 400 \\ 0+\frac{(k-400)}{200}, & 401 \leq k \leq 600 \\ 1+\frac{(k-600)}{400}, & 601 \leq k \leq 1000 .\end{cases}
$$

With this distribution

$$
\Delta x=\left\{\begin{array}{cc}
\frac{1}{400}, & 0 \leq k \leq 399 \\
\frac{1}{200}, & 400 \leq k \leq 599 \\
\frac{1}{400}, & 600 \leq k \leq 999
\end{array}\right.
$$


and

$$
\Delta m=.001 .
$$

Of course our regridding or particle addition algorithm destroys the constancy of $\Delta m$. We regridded whenever $x_{k+1}^{n}-x_{k}^{n} \geq 1 / 100$.

Snapshots of the solution are shown at times $t=1,2,3,4,5$, and 10 in Figures 1-6. By time $t=10$ we have achieved the quasi-steady profile with contact discontinuities at the leading and trailing edge of the traffic and a rarefaction wave in-between. Behind the trailing contact and ahead of the lead contact we have $\rho \equiv 0$. The top figure in each frame shows the density distribution, the middle shows the velocity profile and the bottom figure shows the $\alpha$ profile. To maintain continuity in these graphs we have set $\alpha=0$ and $u=1$ in the regions where $\rho=0$.
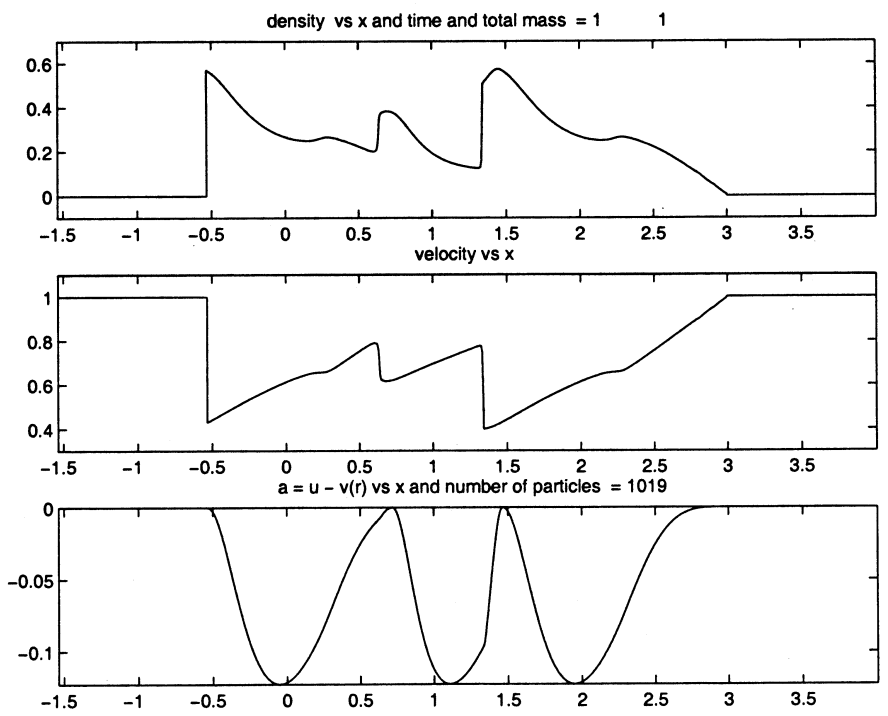

FIG. 1.

The second simulation was run with the periodic initial data

$$
\rho^{0}(x)=.1+.4 \sin ^{2}(\pi x) \text { and } \alpha^{0}=-.2 \sin ^{2}(\pi x) .
$$

This of course yields an initial velocity profile

$$
u^{0}(x)=.9-.6 \sin ^{2}(\pi x) .
$$

Our initial particle positions were

$$
x_{k}^{0}=\frac{k}{400}, \quad-\infty \leq k<\infty,
$$

and we regridded whenever $x_{k+1}^{n}-x_{k}^{n} \geq \frac{1}{200}$. Snapshots of this solution (over four spatial periods) are shown at times $t=.1, .3, .5, .7,2$, and 4 in Figures $7-12$. By this latter time, the solution has converged to a familiar $N$ wave. 

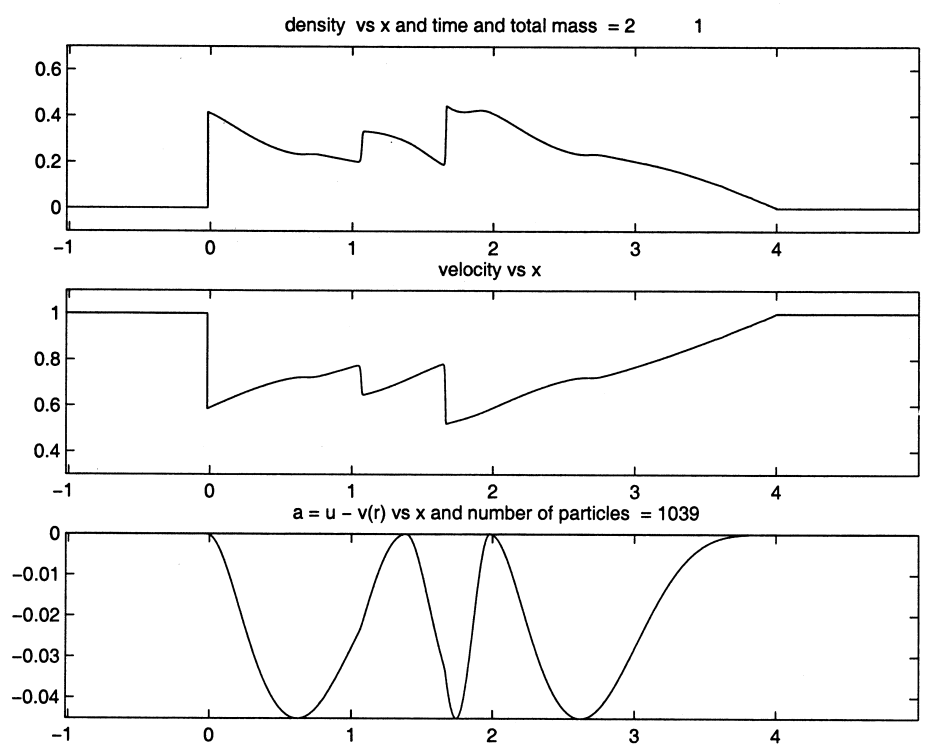

FIG. 2.
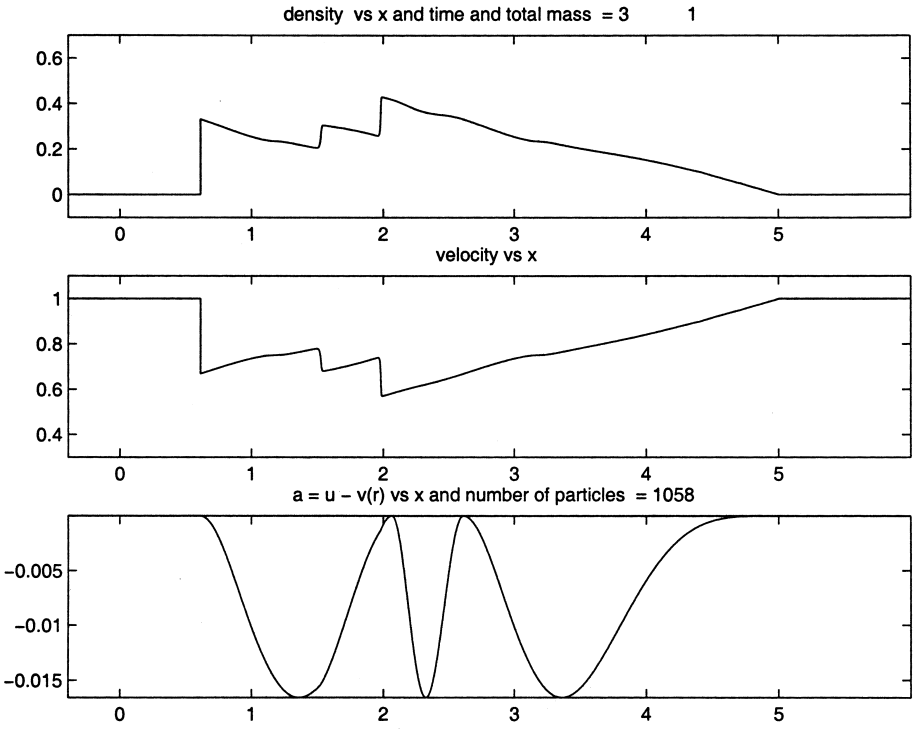

FIG. 3.

Movies of these simulations may be found on the author's web site (http://www. math.cmu.edu/math/people/greenberg.html). Click on traffic1.mpg for the first simulation and traffic2.mpg for the second one. 

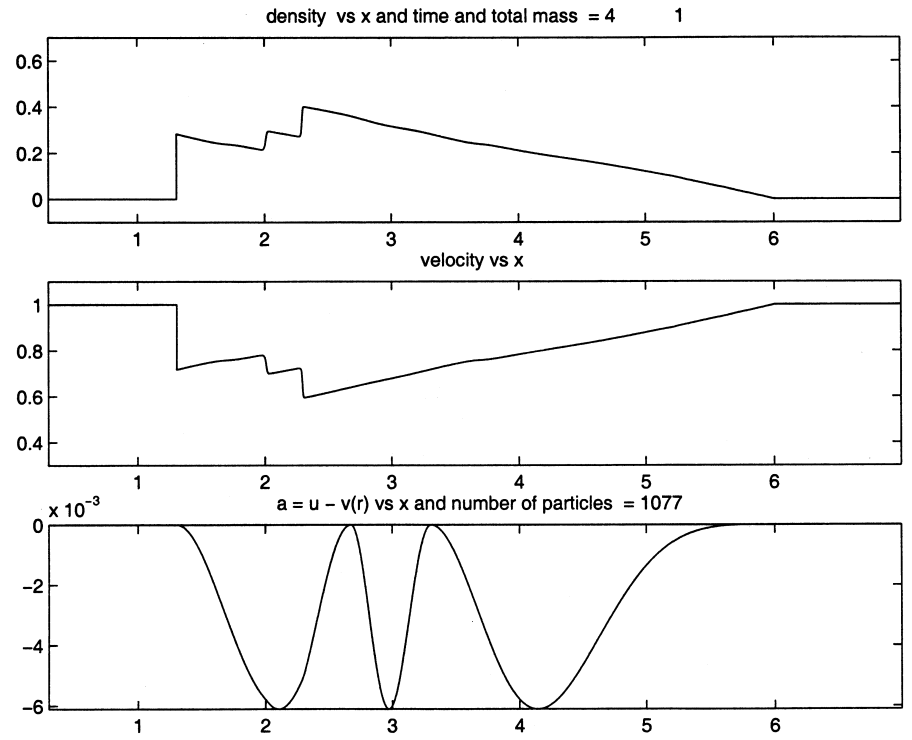

FIG. 4.
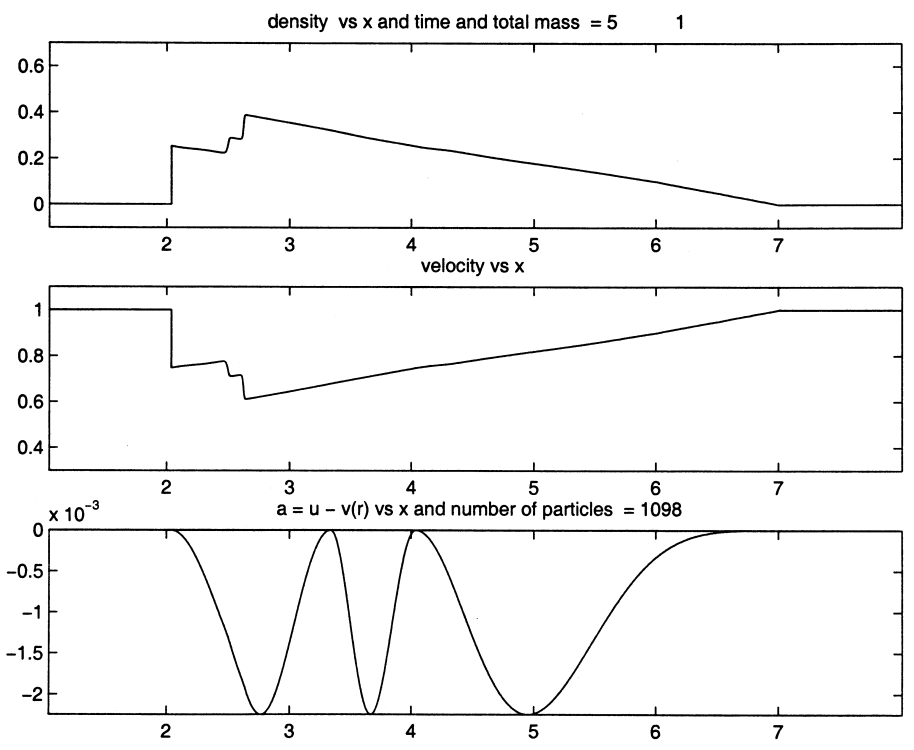

FIG. 5. 

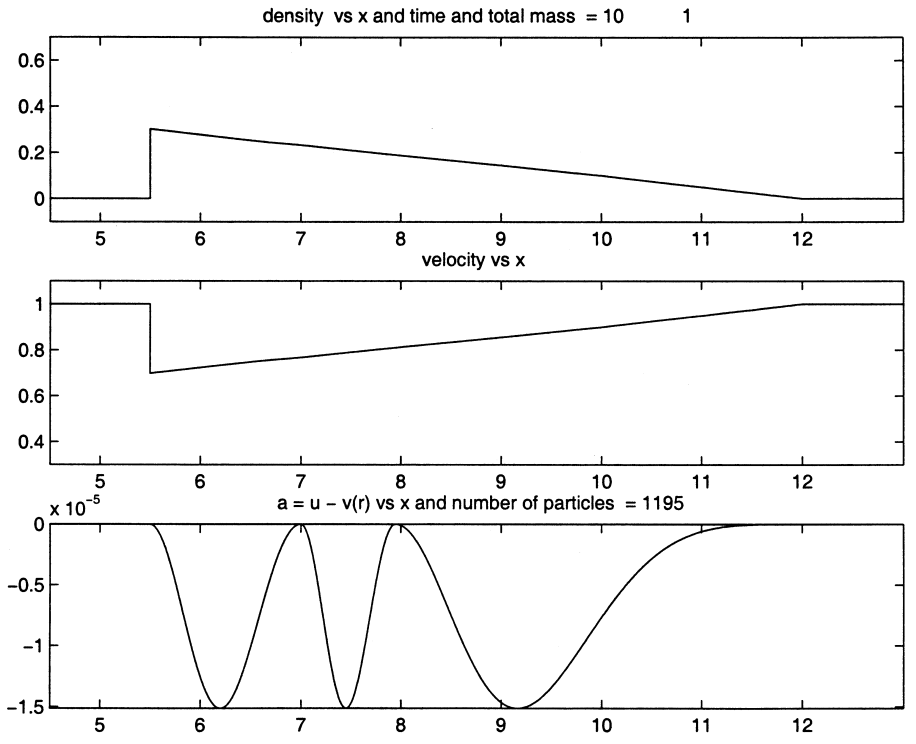

FIG. 6.
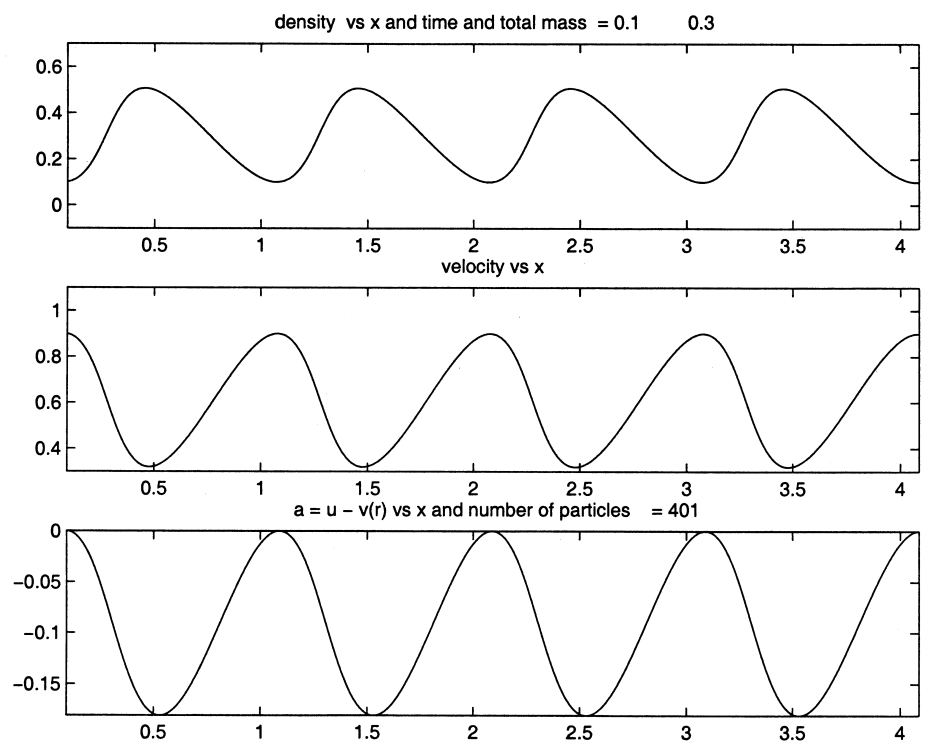

FIG. 7. 

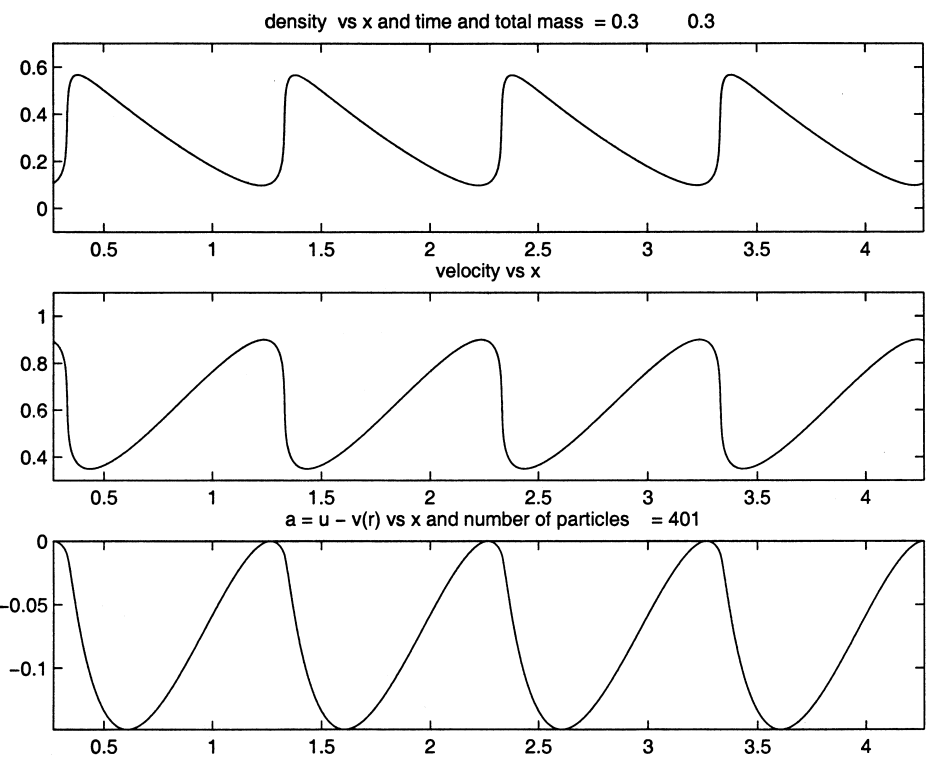

FIG. 8.
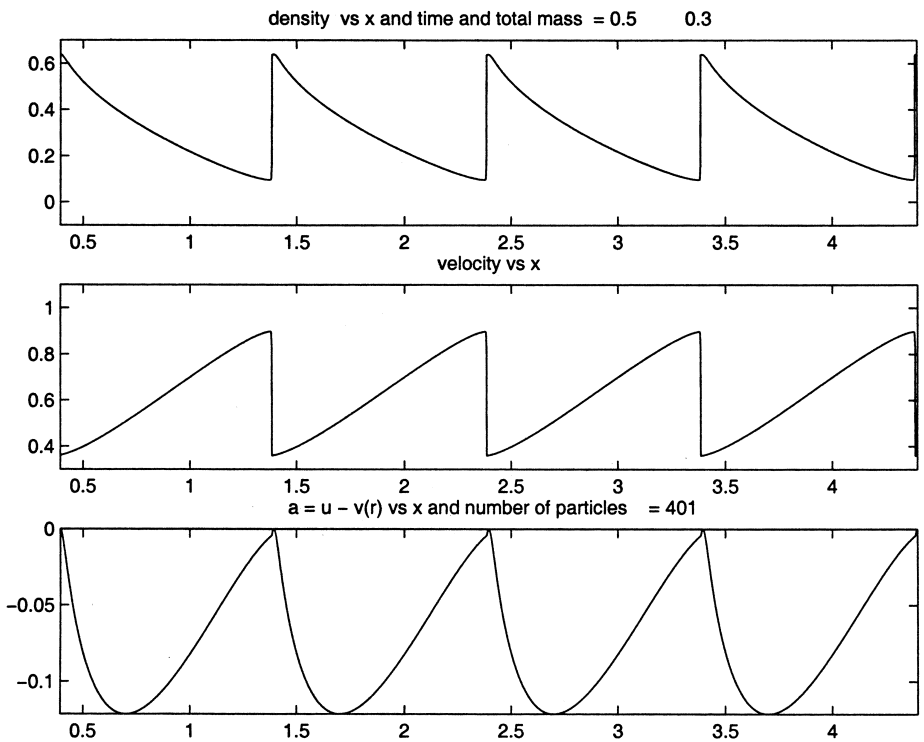

FIG. 9. 

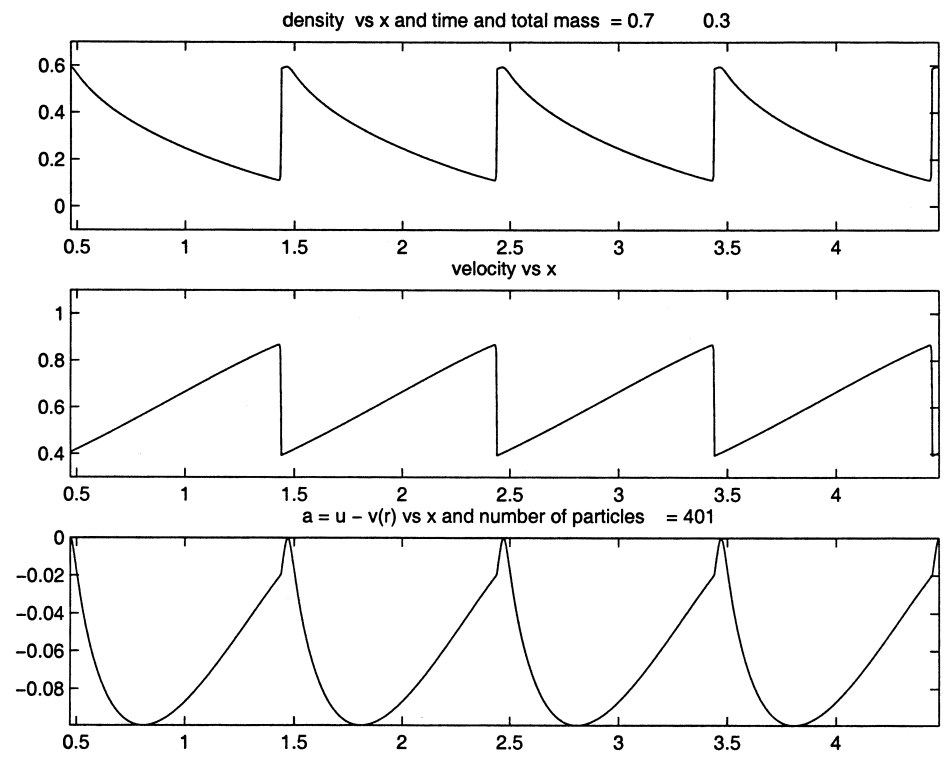

FIG. 10
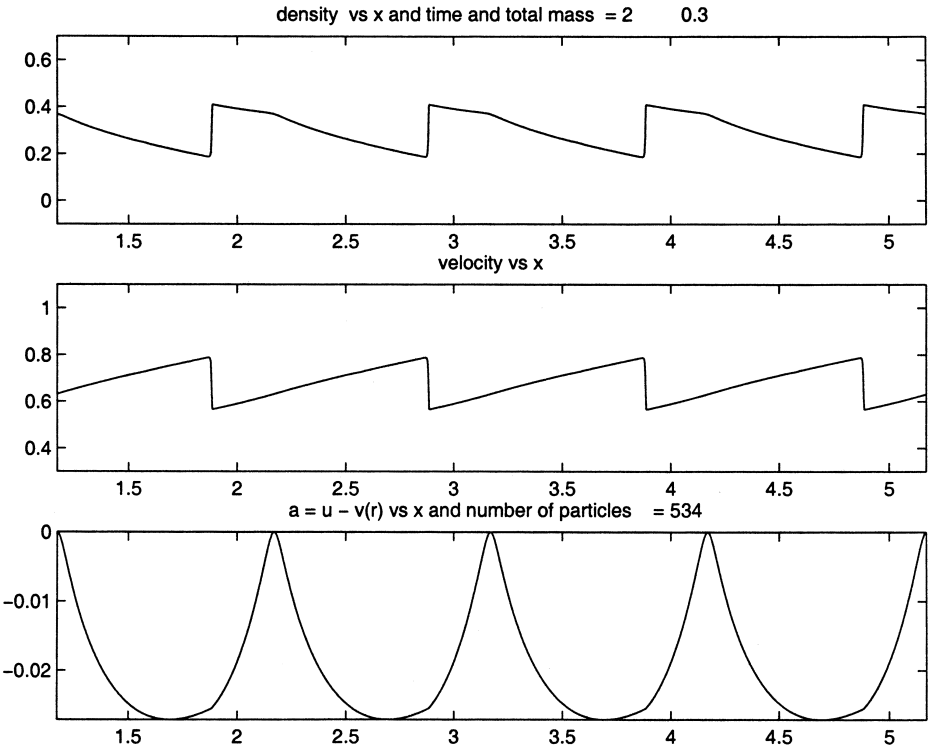

FIG. 11. 

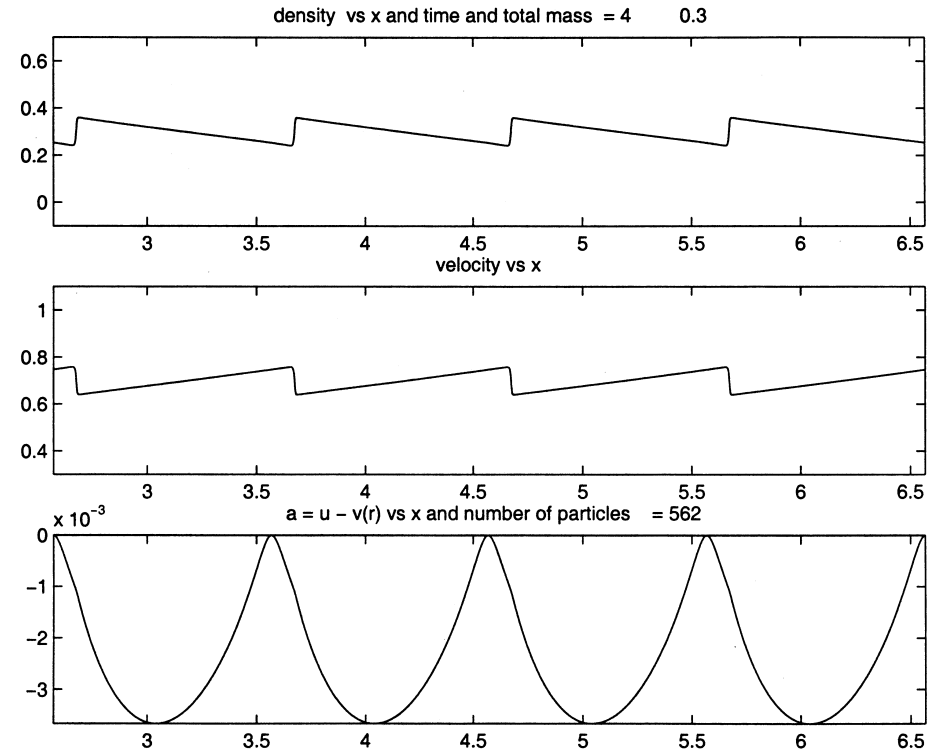

FIG. 12 .

\section{REFERENCES}

[1] A. Aw AND M. RASCle, Resurrection of "second order" models of traffic flow, SIAM J. Appl. Math., 60 (2000), pp. 916-938.

[2] M.J. Lighthill and G.B. Whitham, On kinematic waves. I: Flow movement in long rivers. II: A theory of traffic flow on long crowded roads, Proc. Roy. Soc. London Ser. A, 229 (1955), pp. 281-345.

[3] P.I. Richards, Shock waves on the highway, Oper. Res., 4 (1956), pp. 42-51.

[4] G.B. Whitham, Linear and Nonlinear Waves, Pure Appl. Math., Wiley-Interscience, New York, 1974.

[5] R. Courant and K.O. Friedrichs, Supersonic Flows and Shock Waves, Interscience Publishers, New York, 1948. 